\title{
Endothelium adapts to survive dehydration
}

Medullary renal endothelial cells (mRECs) are exposed to extreme hyperosmolarity in response to dehydration. Now, Peter Carmeliet, Younglun Luo and colleagues show that mRECs metabolically adapt to survive a hyperosmolarity challenge.

"The renal vasculature comprises a wide range of specialized REC phenotypes and our previous work on endothelial cells showed that angiogenesis and cellular quiescence engage specific metabolic pathways," explains Carmeliet. "We therefore aimed to investigate how different RECs adapt to anti-diuresis induced by water deprivation in mice, which exposes mRECs to a hostile environment characterized by extremely high osmolarity that is necessary for urine concentration."

The phenotype of RECs differs throughout the kidney cortex, medulla and glomeruli, as cells in these compartments have different functional requirements and are exposed to unique microenvironments. To further characterize this heterogeneity, the researchers applied single-cell RNA sequencing (scRNA-seq) to mRECs, as wells as RECs from the cortex and glomeruli, from both control

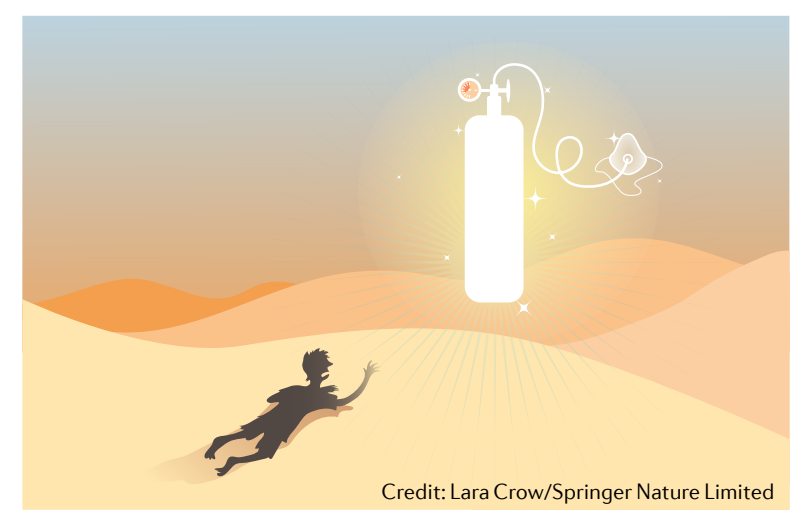

mice under euhydration and mice deprived from drinking water for 12, 24, 36 and 48 hours. "We found a total of 24 REC phenotypes in the healthy mouse kidney, including 8 novel phenotypes," notes Luo. "In addition to the typical quiescent arterial, capillary and venous endothelial cell phenotypes, we identified angiogenic RECs and interferon-activated RECs in healthy adult animals, raising questions about their potential functions in vascular regeneration and immune surveillance, respectively." The protein expression of several transcriptionally enriched markers identified by scRNA-seq was validated by immunostaining.

Acute dehydration results in increased water reabsorption and reduced glomerular filtration in the kidney, leading to water conservation. "RECs responded differentially to water deprivation and mRECs were the most affected, likely due to drastic changes in their microenvironment, including high osmolarity," remarks Carmeliet. Transcriptional changes seemed to occur in two stages - an early adaptation phase (12-24h) and a late response (36-48 h). Early changes included higher expression of heat shock proteins in dehydrated animals than in controls. Transcripts associated with cytoskeleton remodelling, DNA damage and growth arrest were also more highly expressed in dehydrated animals than in controls, and these changes were amplified in the late response. Dehydration also increased the expression of genes linked to cell volume regulation. In contrast to reports on the response of epithelial cells, in mRECs hyperosmolarity reduced the expression of Slc12a2, which encodes a $\mathrm{Na}^{+}-\mathrm{K}^{+}-\mathrm{Cl}^{-}$cotransporter.

Expression of genes associated with cellular responses to hypoxia were higher in mRECs from animals dehydrated for 48 hours than in those from controls, suggesting exposure to higher levels of hypoxia; however, mRECs from these dehydrated animals consumed more oxygen than controls. "Some of the main mREC transcriptional changes in response to dehydration-induced hyperosmotic stress were associated with cell metabolism, including the upregulation of genes linked to glycolysis but also oxidative phosphorylation (OXPHOS)-associated genes - this result was unexpected since endothelial cells are glycolysis-addicted and use glycolysis to produce most of their ATP," observes Carmeliet. "However, OXPHOS generates more ATP per mole of glucose than glycolysis, and this additional ATP could be used for exporting sodium through $\mathrm{Na}^{+}-\mathrm{K}^{+}$ATPases, thus promoting cellular osmolarity equilibration by switching from a toxic rise in intracellular sodium to the accumulation of inert organic osmolytes." Luo adds "metabolic water formation from OXPHOS might also be a protective mechanism against dehydration."

In vitro, the addition of OXPHOS inhibitors to a culture medium in which glucose was replaced with galactose to promote aerobic metabolism compromised the survival of human umbilical vein endothelial cells, but only under hyperosmolar conditions. "Our results emphasize the importance of metabolic plasticity in RECs in response to stress," notes Carmeliet. "The kidney is emerging as a key regulator of systemic metabolism that is as important as the liver in maintaining overall protein and energy homeostasis."

Monica Wang

ORIGINAL ARTICLE Dumas, S. J. et al. Single-cell RNA sequencing reveals renal endothelium heterogeneity and metabolic adaptation to water deprivation.J. Am. Soc. Nephrol. 31, 118-138 (2019) 